\title{
Subcutaneous integrin inhibitors may provide more treatment options for patients with moderate-to-severe ulcerative colitis
}

\author{
Hyuk Yoon \\ Department of Internal Medicine, Seoul National University Bundang Hospital, Seongnam, Korea
}

\begin{abstract}
Article: Efficacy and safety of abrilumab, an $\alpha 4 \beta 7$ integrin inhibitor, in Japanese patients with moderate-to-severe ulcerative colitis: a phase II study (Intest Res 2019;17:375-386)
\end{abstract}

Vedolizumab binds to $\alpha 4 \beta 7$ integrin, which is specifically expressed by a subset of gastrointestinal-homing T lymphocytes, and prevents $\alpha 4 \beta 7$ integrin from binding to mucosal addressin cell adhesion molecule-1 in mucosal endothelial cells. ${ }^{1}$ The efficacy and safety of this gut-selective biologic in UC were confirmed in clinical studies ${ }^{2,3}$ and with real-world data. ${ }^{4}$ However, because vedolizumab is administered intravenously, it is not convenient for some patients. Abrilumab is another integrin inhibitor that selectively targets $\alpha 4 \beta 7$ dimer. It is extensively absorbed after subcutaneous dosing, and it has a high bioavailability and a long half-life. ${ }^{5}$ The efficacy and safety of abrilumab for induction therapy have been recently reported in western patients with moderate-to-severe UC. ${ }^{6}$ In this phase $2 \mathrm{~b}$ study, patients were randomized in a $2: 1: 2: 2: 2$ ratio to subcutaneous placebo or abrilumab at $7 \mathrm{mg}, 21 \mathrm{mg}, 70 \mathrm{mg}$ (on day 1 , week 2 , week 4 , and every 4 weeks thereafter until week 24 ), or $210 \mathrm{mg}$ (on day 1 followed by placebo in weeks 2 and 4 and every 4 weeks thereafter until week 24), respectively. Abrilumab resulted in significantly greater remission, response, and

Received June 7, 2019. Accepted June 9, 2019.

Correspondence to Hyuk Yoon, Department of Internal Medicine, Seoul

National University Bundang Hospital, 82 Gumi-ro 173beon-gil, Bundang-

gu, Seongnam 13620, Korea. Tel: +82-31-787-7042, Fax: +82-31-787-4051,

E-mail: bodnsoul@hanmail.net

ORCID Hyuk Yoon (https://orcid.org/0000-0002-2657-0349) mucosal healing rates than the placebo. The remission rates at week 8 were $4.3 \%, 13.3 \%$, and $12.7 \%$ for the placebo, abrilumab 70-mg, and abrilumab 210-mg groups, respectively $(P<0.05$ for the 70-mg and 210-mg groups vs. placebo group). These results were similar to those observed in a phase 3 GEMINI I trial of vedolizumab in UC patients. ${ }^{2}$

In the present issue of Intestinal Research, Hibi et al. ${ }^{7}$ reported the results of another phase 2 study involving abrilumab in Japanese UC patients. Forty-five UC patients were randomized to $21 \mathrm{mg}(\mathrm{n}=11), 70 \mathrm{mg}(\mathrm{n}=12)$, or $210 \mathrm{mg}(\mathrm{n}=9)$ of abrilumab, or placebo $(\mathrm{n}=13)$ via subcutaneous injection for 12 weeks. The primary endpoint was clinical remission at week 8 (total Mayo score $\leq 2$ points with no individual subscore $>1$ point). The double-blind period was followed by a 36-week open-label period, in which all patients received abrilumab 210 mg subcutaneously every 12 weeks, and a 28-week safety follow-up period. Clinical remission at week 8 was 4 out of 31 (12.9\%) overall in the abrilumab groups versus 0 out of 13 in the placebo group (abrilumab: $21 \mathrm{mg}, 1 / 10$ [10.0\%]; $70 \mathrm{mg}$, 2/12 [16.7\%]; $210 \mathrm{mg}, 1 / 9$ [11.1\%]). In both the double-blind and open-label periods, more patients in the placebo group experienced $\geq 1$ adverse event compared to those in the abrilumab groups.

In this study, although the results were not statistically significant, abrilumab $70 \mathrm{mg}$ and $210 \mathrm{mg}$ yielded numerically 
better results in terms of clinical remission rate at week 8 than placebo. In addition, the clinical remission rates for the 70-mg abrilumab dose were higher than those reported in a previous study conducted in North America, Europe, and Australia (16.7\% vs. $13.5 \%) .{ }^{6}$ The clinical response rates at week 8 for abrilumab $70 \mathrm{mg}$ and $210 \mathrm{mg}$ in this study (50.0\% and 66.7\%) were similar to those in the aforementioned study $(49.0 \%$ and $46.8 \%)$, even though the response rate of the placebo group in the present study was strangely higher than that in the aforementioned study ( $53.8 \%$ vs. $25.9 \%$ ). Therefore, the present study suggests that abrilumab might be effective and tolerable in Japanese patients with moderate-to-severe UC similar to western patients. However, the statistical power of the present study was too low to detect the true efficacy of abrilumab in UC patients. In addition, evaluating the efficacy of abrilumab according to prior usage of an anti-TNF- $\alpha$ inhibitor was not feasible due to the small sample size in the present study. Therefore, further studies in a larger number of Asian UC patients are required.

Other trials to assess the efficacy of the integrin inhibitor administered subcutaneously in UC patients are in progress. The preliminary results of the VISIBLE 1 trial, which evaluated the efficacy of a bi-weekly subcutaneous vedolizumab administration as a maintenance therapy in UC patients who showed a clinical response following intravenous vedolizumab induction therapy, were recently reported. ${ }^{8}$ A statistically significant proportion of patients who received vedolizumab subcutaneously achieved clinical remission compared to those who received the placebo ( $46.2 \%$ vs. $14.3 \%$; $P<0.001)$ at week 52 . A similar rate of clinical remission was observed in the vedolizumab intravenous reference arm $(42.6 \%)$ at week 52 . Several phase 3 trials to evaluate the efficacy of etrolizumab, another subcutaneous integrin inhibitor, are also underway in patients with UC. ${ }^{9}$ Subcutaneous integrin inhibitors are expected to provide more treatment options for patients with moderate-tosevere UC. We are eagerly awaiting the results of these trials.

\section{FINANCIAL SUPPORT}

The authors received no financial support for the research, authorship, and/or publication of this article.

\section{CONFLICT OF INTEREST}

No potential conflict of interest relevant to this article was reported.

\section{AUTHOR CONTRIBUTION}

Writing and approval of final manuscript: Yoon $\mathrm{H}$.

\section{REFERENCES}

1. Wyant T, Fedyk E, Abhyankar B. An overview of the mechanism of action of the monoclonal antibody vedolizumab. J Crohns Colitis 2016;10:1437-1444.

2. Feagan BG, Rutgeerts P, Sands BE, et al. Vedolizumab as induction and maintenance therapy for ulcerative colitis. N Engl J Med 2013;369:699-710.

3. Loftus EV Jr, Colombel JF, Feagan BG, et al. Long-term efficacy of vedolizumab for ulcerative colitis. J Crohns Colitis 2017;11: 400-411.

4. Baumgart DC, Bokemeyer B, Drabik A, Stallmach A, Schreiber S; Vedolizumab Germany Consortium. Vedolizumab induction therapy for inflammatory bowel disease in clinical practice: a nationwide consecutive German cohort study. Aliment Pharmacol Ther 2016;43:1090-1102.

5. Pan WJ, Köck K, Rees WA, et al. Clinical pharmacology of AMG 181, a gut-specific human anti-alpha4beta7 monoclonal antibody, for treating inflammatory bowel diseases. Br J Clin Pharmacol 2014;78:1315-1333.

6. Sandborn WJ, Cyrille M, Hansen MB, et al. Efficacy and safety of abrilumab in a randomized, placebo-controlled trial for moderate-to-severe ulcerative colitis. Gastroenterology 2019; 156:946-957.

7. Hibi T, Motoya S, Ashida T, et al. Efficacy and safety of abrilumab, an alpha4beta7 integrin inhibitor, in Japanese patients with moderate-to-severe ulcerative colitis: a phase II study. Intest Res 2019;17;375-386.

8. Sandborn WJ, Baert F, Danese S, et al. Efficacy and safety of a new vedolizumab subcutaneous formulation for ulcerative colitis: results of the VISIBLE 1 phase 3 trial. United European Gastroenterology J 2018;6(Suppl 1).

9. Pérez-Jeldres T, Tyler CJ, Boyer JD, et al. Cell trafficking interference in inflammatory bowel disease: therapeutic interventions based on basic pathogenesis concepts. Inflamm Bowel Dis 2019;25:270-282. 